\title{
Language Learning Strategies Applied by Arabic Learners: A Case-Study at Institute of Education, International Islamic University Malaysia (IIUM)
}

\author{
Dr. ArifinMamat,IbrohemSideh \\ 1 Institute of Education, International Islamic University Malaysia (IIUM) \\ 2 Institute of Education, International Islamic University Malaysia (IIUM)
}

\begin{abstract}
Language learning strategies (LLS) play an important role in a second/foreign language acquisition, as they promote and facilitate language learning. Thisstudy aims to investigate LLS used by studentsin learning the Arabic language. The respondents of the study were all final year students at the Institute of Education, the International Islamic University of Malaysia (IIUM). Strategy Inventory for Language Learning (SILL) developed by Oxford (1990) was used to gather the data for the study. While, a simple descriptive statistical analysis was employed to provide meanings to the data. It includes frequency distribution, percentages, means, standard deviation and range of scores for the data set. The studyreveals thatstudents were familiar with entire six LLS categories namely; memory, cognitive, compensation, metacognitive, affective, and social strategies, with a medium frequency level use for overall strategies $(M=3.49)$. Among these six strategies, the most frequently used from the top was metacognitive, compensation, social,memory, cognitive, and affective strategies.
\end{abstract}

Keywords:Learning strategies, language learning strategies (LLS), learning Arabic as a second/foreign language (ASL/AFL), second/foreign language acquisition, teaching Arabic

\section{Introduction}

Language learning strategies (LLS) play the key roles in a second/foreign language acquisition, as they promote and facilitate language learning. Due to this matter, research into LLS has been increased and burgeoned significantly in the 1970s with the cognitive revolution. A number of studies have revealed a close relationship between LLS and language achievement. Oxford describes LLS as "specific action taken by the learner to make learning easier, faster, more enjoyable, more self-directed, more effective and more transferable to new situations" (1990: 8). She also maintains that learning strategies play a significant role for language learners to improve the development of their second or foreign language learning skills. In addition, to be effective in learning a foreign language, learners must be conscious of using sufficient learning strategies (Meshyan\& Hernandez, 2002; Sueraya, Ismail, Arifin\&Ismaiel, 2010).As a result, not having suitable language learning strategies becomes an obstacle for students to improve their second language.

Several studies also suggest that learning strategies are able to assist learners in elevating their own accomplishment in language proficiency (Bremner, 1998; O'Malley, 1985; Politzer, 1983). Anida's (2003) study that has successfully verified that students are able to improve their performance, self-confidence, interests, and attitude towards the Arabic language by relying on the strategies used further indicates a link between LLS and language proficiency. In this case, the differences between more and less successful language learners have been associated with the amount and choice of strategies used, and how suitable strategies are applied to the task.

Nevertheless, a great number of Arabic learners viewed learning Arabic language as too complicated and time consuming to acquire, as found in several studies (i.e., Burk, 2005; Duereh, 1999; Doka, 2000; Etae, S. 2009; Hobrom, 2004; Qais, Zainab\&Hamidah, 2007; Wan Nordin, 2006).

Therefore this study attempts to investigate Language Learning Strategies (LLS) used by students in learning the Arabic language and identify the most frequent strategies applied by them.

The extent of using LLS categories in this study is based on Oxford Taxonomy. She divides LLS into two major classifications: direct and indirect strategies, which are further subdivided into a total of six groups. The direct strategies consisted of memory, cognitive, and compensation strategies. The indirect strategies on the other side contained of metacognitive, affective, and social strategies (Oxford, 1990).

\section{Research Methodology}

The researcher in this study adopted the survey method, which is widely used in educational research. Survey research designs are procedures in quantitative research in which investigators and administer survey to 
a sample or to the entire population of people in order to describe the attitudes, opinions, behaviors, or characteristics of the population (Creswell, 2008).

\subsection{Population and Sample}

The population and sample to which the results of the study were generalized comprised students who study Bachelor of Education majoring in the Teaching of Arabic as a Second language at the Institute of Education, the International Islamic University of Malaysia. There are thirty students (30). The questionnaires were distributed to all of them.

\section{Iii. Scale}

This study utilizes the questionnaire as an instrument of gathering the data and to answer the objectives of the study. It was divided into two sections. The first was the demographic background of participants (3 items). The second was Strategy Inventory for Language Learning (SILL), which was developed by Oxford (1990). It was used to investigate the type and frequency of strategies practiced by the participants (48 items). These 48 items were based on six different strategy categories use namely; memory strategies (9items), cognitive strategies (11 items), compensation strategies (6 items), metacognitive strategies (9 items), affective strategies (6 items), and social strategies ( 7 items). Also, the scoring scale for all 48 items in SILL questionnaire were raged by using 5-likert scale such 1 indicated "Never or almost never true of me" (Never), 2 indicated "Generally not true of me" (Not true), 3 indicated "Somewhat true of me" (Somewhat), 4 indicated "Generally true of me" (True), and 5 indicated "Always or almost always true of me" (Always).

\subsection{Data Analysis}

All responses on the returned questionnaires were tabulated in numerical code for subsequent analyses. Data from the questionnaires were coded and entered into a computer database using a software package, the Statistical Package for Social Sciences (SPSS) to analyze and manage quantitative data for this study.

A descriptive analysis of all variables was conducted to provide meanings to the data. Descriptive analysis provides simple summaries and general information about the data set of the study where the researcher could obtain descriptive statistics such as frequency distribution, percentages, means, standard deviation and range of scores for the data set. Therefore, the researcher would be able to understand the level of the distribution and its spread (Robson, 1997).

\section{Research Findings}

Table 1

Memory strategies

\begin{tabular}{|c|c|c|c|c|c|c|c|}
\hline $\begin{array}{l}\text { Item } \\
\text { No. }\end{array}$ & Statement & $\begin{array}{c}\text { Never } \\
\% \\
(\mathbf{N})\end{array}$ & $\begin{array}{l}\text { Not true } \\
\% \\
\text { (N) }\end{array}$ & $\begin{array}{l}\text { Somewhat } \\
\% \\
\text { (N) }\end{array}$ & $\begin{array}{c}\text { True } \\
\% \\
(\mathbf{N})\end{array}$ & $\begin{array}{c}\text { Always } \\
\% \\
\text { (N) }\end{array}$ & $\mathbf{M}$ \\
\hline 1 & $\begin{array}{l}\text { Memory Strategies: } \\
\text { I review Arabic lessons often. }\end{array}$ & $\begin{array}{l}3.3 \\
(1)\end{array}$ & - & $\begin{array}{l}73.3 \\
(22)\end{array}$ & $\begin{array}{l}23.3 \\
(7)\end{array}$ & - & 3.16 \\
\hline 2 & I use rhymes to remember Arabic words. & $\begin{array}{l}3.3 \\
(1)\end{array}$ & $\begin{array}{l}10.0 \\
(3)\end{array}$ & $\begin{array}{l}63.3 \\
(19)\end{array}$ & $\begin{array}{c}23.3 \\
(7)\end{array}$ & - & 3.06 \\
\hline 3 & $\begin{array}{l}\text { I use flashcards to remember new Arabic } \\
\text { words. }\end{array}$ & $\begin{array}{c}13.3 \\
(4)\end{array}$ & $\begin{array}{l}26.7 \\
(8)\end{array}$ & $\begin{array}{l}43.3 \\
(13)\end{array}$ & $\begin{array}{l}16.7 \\
(5)\end{array}$ & - & 2.63 \\
\hline 4 & I physically act out new Arabic words. & - & $\begin{array}{l}16.7 \\
(5)\end{array}$ & $\begin{array}{l}43.3 \\
(13)\end{array}$ & $\begin{array}{l}36.7 \\
(11)\end{array}$ & $\begin{array}{l}3.3 \\
(1)\end{array}$ & 3.26 \\
\hline 5 & $\begin{array}{l}\text { I think of relationships between what I } \\
\text { already know and new things I learn in } \\
\text { Arabic. }\end{array}$ & - & $\begin{array}{l}3.3 \\
(1)\end{array}$ & $\begin{array}{l}13.3 \\
(4)\end{array}$ & $\begin{array}{l}70.0 \\
(21)\end{array}$ & $\begin{array}{c}13.3 \\
(4)\end{array}$ & 3.93 \\
\hline 6 & $\begin{array}{l}\text { I use new Arabic words in a sentence so I } \\
\text { can remember them. }\end{array}$ & - & $\begin{array}{l}6.7 \\
(2)\end{array}$ & $\begin{array}{c}23.3 \\
(7)\end{array}$ & $\begin{array}{l}60.0 \\
(18)\end{array}$ & $\begin{array}{l}10.0 \\
(3)\end{array}$ & 3.73 \\
\hline 7 & $\begin{array}{l}\text { I remember a new Arabic word by } \\
\text { making a mental picture of a situation in } \\
\text { which the word might be used. }\end{array}$ & $\begin{array}{l}3.3 \\
(1)\end{array}$ & $\begin{array}{l}10.0 \\
(3)\end{array}$ & $\begin{array}{l}26.7 \\
(8)\end{array}$ & $\begin{array}{l}50.0 \\
(15)\end{array}$ & $\begin{array}{l}10.0 \\
(3)\end{array}$ & 3.53 \\
\hline 8 & $\begin{array}{l}\text { I remember new Arabic words or phrases } \\
\text { by remembering their location on the } \\
\text { page, on the board, or on a street sign. }\end{array}$ & - & $\begin{array}{l}13.3 \\
(4)\end{array}$ & $\begin{array}{l}16.7 \\
(5)\end{array}$ & $\begin{array}{l}56.7 \\
(17)\end{array}$ & $\begin{array}{l}13.3 \\
(4)\end{array}$ & 3.70 \\
\hline 9 & $\begin{array}{l}\text { I connect the sound of a new Arabic } \\
\text { word and image or picture of the word to } \\
\text { help me remember the word }\end{array}$ & - & $\begin{array}{l}13.3 \\
(4)\end{array}$ & $\begin{array}{l}33.3 \\
(10)\end{array}$ & $\begin{array}{l}36.7 \\
(11)\end{array}$ & $\begin{array}{c}16.7 \\
(5)\end{array}$ & 3.56 \\
\hline
\end{tabular}


Table 1 above indicated that the utilization of memory strategies by the participants in learning Arabic fell within the mean score of 2.63-3.93. In fact, the strategy "thinking of relationships between what is already known and new things are currently learnt in Arabic" (item 5) was the most frequently used by them, holding the mean of 3.93. While, the least frequently used was the strategy "using flashcards to remember new Arabic words" (item 3), where its mean score carried only 2.63 .

Table 2

Cognitive strategies

\begin{tabular}{|c|c|c|c|c|c|c|c|}
\hline $\begin{array}{l}\text { Item } \\
\text { No. }\end{array}$ & Statement & $\begin{array}{c}\text { Never } \\
\% \\
(\mathrm{~N})\end{array}$ & $\begin{array}{c}\text { Not true } \\
\% \\
(\mathrm{~N}) \\
\end{array}$ & $\begin{array}{c}\text { Somewhat } \\
\% \\
(\mathbf{N}) \\
\end{array}$ & $\begin{array}{c}\text { True } \\
\% \\
(\mathrm{~N})\end{array}$ & $\begin{array}{c}\text { Always } \\
\% \\
\text { (N) }\end{array}$ & M \\
\hline 10 & $\begin{array}{l}\text { Cognitive Strategies: } \\
\text { I read for pleasure in Arabic. }\end{array}$ & - & $\begin{array}{l}10.0 \\
(3)\end{array}$ & $\begin{array}{c}23.3 \\
(7)\end{array}$ & $\begin{array}{l}56.7 \\
(17)\end{array}$ & $\begin{array}{c}10.0 \\
(3)\end{array}$ & 3.66 \\
\hline 11 & I practice the sounds in Arabic. & - & $\begin{array}{l}10.0 \\
(3)\end{array}$ & $\begin{array}{c}30.0 \\
(9)\end{array}$ & $\begin{array}{l}53.3 \\
(16)\end{array}$ & $\begin{array}{l}6.7 \\
(2)\end{array}$ & 3.56 \\
\hline 12 & I start conversations in Arabic. & $\begin{array}{l}3.3 \\
(1)\end{array}$ & $\begin{array}{c}20.0 \\
(6)\end{array}$ & $\begin{array}{l}33.3 \\
(10)\end{array}$ & $\begin{array}{l}40.0 \\
(12)\end{array}$ & $\begin{array}{l}3.3 \\
(1)\end{array}$ & 3.20 \\
\hline 13 & I try to talk like native Arabic speakers. & - & $\begin{array}{l}20.0 \\
(6)\end{array}$ & $\begin{array}{c}30.0 \\
(9)\end{array}$ & $\begin{array}{l}36.7 \\
(11)\end{array}$ & $\begin{array}{c}13.3 \\
(4)\end{array}$ & 3.43 \\
\hline 14 & $\begin{array}{l}\text { I write notes, messages, letters or } \\
\text { reports in Arabic. }\end{array}$ & $\begin{array}{l}3.3 \\
(1)\end{array}$ & $\begin{array}{l}20.0 \\
(6)\end{array}$ & $\begin{array}{l}46.7 \\
(14)\end{array}$ & $\begin{array}{c}30.0 \\
(9)\end{array}$ & - & 3.03 \\
\hline 15 & $\begin{array}{l}\text { I use the Arabic words I know in } \\
\text { different ways. }\end{array}$ & - & $\begin{array}{l}6.7 \\
(2)\end{array}$ & $\begin{array}{l}33.3 \\
(10)\end{array}$ & $\begin{array}{l}60.0 \\
(18)\end{array}$ & - & 3.53 \\
\hline 16 & I try not to translate word-for word. & - & $\begin{array}{l}13.3 \\
(4)\end{array}$ & $\begin{array}{l}33.3 \\
(10)\end{array}$ & $\begin{array}{l}43.3 \\
(13)\end{array}$ & $\begin{array}{l}10.0 \\
(3)\end{array}$ & 3.50 \\
\hline 17 & $\begin{array}{l}\text { I say or write new Arabic words } \\
\text { several times. }\end{array}$ & - & $\begin{array}{l}6.7 \\
(2)\end{array}$ & $\begin{array}{l}36.7 \\
(11)\end{array}$ & $\begin{array}{l}53.3 \\
(16)\end{array}$ & $\begin{array}{l}3.3 \\
(1)\end{array}$ & 3.53 \\
\hline 18 & $\begin{array}{l}\text { I first skim in Arabic passage then go } \\
\text { back and read carefully. }\end{array}$ & $\begin{array}{l}3.3 \\
(1)\end{array}$ & $\begin{array}{l}3.3 \\
(1)\end{array}$ & $\begin{array}{l}36.7 \\
(11)\end{array}$ & $\begin{array}{l}40.0 \\
(12)\end{array}$ & $\begin{array}{l}16.7 \\
(5)\end{array}$ & 3.63 \\
\hline 19 & $\begin{array}{l}\text { I watch Arabic language TV shows } \\
\text { spoken in Arabic or go to movies } \\
\text { spoken in Arabic. }\end{array}$ & $\begin{array}{c}16.7 \\
(5)\end{array}$ & $\begin{array}{l}33.3 \\
(10)\end{array}$ & $\begin{array}{c}26.7 \\
(8)\end{array}$ & $\begin{array}{c}23.3 \\
(7)\end{array}$ & - & 2.56 \\
\hline 20 & $\begin{array}{l}\text { I make summaries of information that I } \\
\text { hear or read in Arabic. }\end{array}$ & $\begin{array}{l}3.3 \\
(1)\end{array}$ & $\begin{array}{c}13.3 \\
(4)\end{array}$ & $\begin{array}{l}40.0 \\
(12)\end{array}$ & $\begin{array}{l}43.3 \\
(13)\end{array}$ & - & 3.23 \\
\hline
\end{tabular}

With regard to the results of using cognitive strategies, Table 2 exhibited that the mean score of strategy "reading for pleasure in Arabic" (item 10) was observed to be the highest with 3.66 whereas, the lowest mean (2.56) was appertained to the strategy "watching Arabic language TV shows spoken in Arabic or going to movies spoken in Arabic" (item 19).

Table 3

Compensation strategies

\begin{tabular}{|c|c|c|c|c|c|c|c|}
\hline $\begin{array}{c}\text { Item } \\
\text { No. }\end{array}$ & Statement & $\begin{array}{c}\text { Never } \\
\% \\
(\mathbf{N})\end{array}$ & $\begin{array}{l}\text { Not true } \\
\% \\
\text { (N) }\end{array}$ & $\begin{array}{c}\text { Somewhat } \\
\% \\
\text { (N) }\end{array}$ & $\begin{array}{c}\text { True } \\
\% \\
(\mathbf{N})\end{array}$ & $\begin{array}{c}\text { Always } \\
\% \\
\text { (N) }\end{array}$ & $\mathbf{M}$ \\
\hline 21 & $\begin{array}{l}\text { Compensation strategies: } \\
\text { I read Arabic without looking up every } \\
\text { new words. }\end{array}$ & $\begin{array}{c}13.3 \\
(4)\end{array}$ & $\begin{array}{l}30.0 \\
(9)\end{array}$ & $\begin{array}{c}23.3 \\
(7)\end{array}$ & $\begin{array}{l}33.3 \\
(10)\end{array}$ & - & 2.76 \\
\hline 22 & $\begin{array}{l}\text { I try to guess what other person will say } \\
\text { next in Arabic }\end{array}$ & $\begin{array}{l}3.3 \\
(1)\end{array}$ & $\begin{array}{l}10.0 \\
(3)\end{array}$ & $\begin{array}{l}30.0 \\
(9)\end{array}$ & $\begin{array}{l}50.0 \\
(15)\end{array}$ & $\begin{array}{l}6.7 \\
(2)\end{array}$ & 3.46 \\
\hline 23 & $\begin{array}{l}\text { To understand unfamiliar Arabic words, } \\
\text { I make guesses. }\end{array}$ & - & - & $\begin{array}{c}23.3 \\
(7)\end{array}$ & $\begin{array}{l}63.3 \\
(19)\end{array}$ & $\begin{array}{l}13.3 \\
(4)\end{array}$ & 3.90 \\
\hline 24 & $\begin{array}{l}\text { When I can't think of a word during a } \\
\text { conversation in Arabic, I use gestures. }\end{array}$ & - & $\begin{array}{l}3.3 \\
(1)\end{array}$ & $\begin{array}{l}16.7 \\
(5)\end{array}$ & $\begin{array}{l}60.0 \\
(18)\end{array}$ & $\begin{array}{c}20.0 \\
(6)\end{array}$ & 3.96 \\
\hline 25 & $\begin{array}{l}\text { If I can't think of an Arabic word, I use a } \\
\text { word or phrase that means the same } \\
\text { thing. }\end{array}$ & - & $\begin{array}{l}10.0 \\
\text { (3) }\end{array}$ & $\begin{array}{l}16.7 \\
(5)\end{array}$ & $\begin{array}{l}40.0 \\
(12)\end{array}$ & $\begin{array}{l}33.3 \\
(10)\end{array}$ & 3.96 \\
\hline 26 & $\begin{array}{l}\text { I make up new words if I do not know } \\
\text { the right ones in Arabic. }\end{array}$ & - & $\begin{array}{l}10.0 \\
(3)\end{array}$ & $\begin{array}{l}36.7 \\
(11)\end{array}$ & $\begin{array}{l}36.7 \\
(11)\end{array}$ & $\begin{array}{l}16.7 \\
(5)\end{array}$ & 3.60 \\
\hline
\end{tabular}

Based on the statistical data for compensation strategies revealed in table 3 above, it showed that the greatest mean score (3.96) was shared by the strategy "using gestures when unable to think of a word during a conversation in Arabic" (item 24) and the strategy "using a word or phrase that means the same thing if unable to think of an Arabic word" (item 25). The strategy "reading Arabic without looking up every new words" (item 21 ) on the other hand, carried the smallest mean of 2.76 . 
Table 4

Metacognitive strategies

\begin{tabular}{|c|c|c|c|c|c|c|c|}
\hline $\begin{array}{l}\text { Item } \\
\text { No. }\end{array}$ & Statement & $\begin{array}{l}\text { Never } \\
\% \\
\text { (N) }\end{array}$ & $\begin{array}{c}\text { Not true } \\
\% \\
\text { (N) }\end{array}$ & $\begin{array}{c}\text { Somewhat } \\
\% \\
\text { (N) }\end{array}$ & $\begin{array}{c}\text { True } \\
\% \\
(\mathrm{~N})\end{array}$ & $\begin{array}{c}\text { Always } \\
\% \\
\text { (N) }\end{array}$ & M \\
\hline 27 & $\begin{array}{l}\text { Metacognitive strategies: } \\
\text { I pay attention when someone is } \\
\text { speaking Arabic. }\end{array}$ & - & - & $\begin{array}{c}10.0 \\
(3)\end{array}$ & $\begin{array}{l}63.3 \\
(19)\end{array}$ & $\begin{array}{c}26.7 \\
(8)\end{array}$ & 4.16 \\
\hline 28 & $\begin{array}{l}\text { I try to find as many ways as I can to use } \\
\text { my Arabic. }\end{array}$ & - & $\begin{array}{l}13.3 \\
(4)\end{array}$ & $\begin{array}{l}33.3 \\
(10)\end{array}$ & $\begin{array}{c}30.0 \\
(9)\end{array}$ & $\begin{array}{c}23.3 \\
(7)\end{array}$ & 3.63 \\
\hline 29 & $\begin{array}{l}\text { I think about my progress in learning } \\
\text { Arabic. }\end{array}$ & - & $\begin{array}{l}6.7 \\
(2)\end{array}$ & $\begin{array}{c}16.7 \\
(5)\end{array}$ & $\begin{array}{l}46.7 \\
(14)\end{array}$ & $\begin{array}{c}30.0 \\
(9)\end{array}$ & 4.00 \\
\hline 30 & I look for people I can talk to in Arabic. & $\begin{array}{l}3.3 \\
(1)\end{array}$ & $\begin{array}{l}13.3 \\
(4)\end{array}$ & $\begin{array}{l}36.7 \\
(11)\end{array}$ & $\begin{array}{c}30.0 \\
(9)\end{array}$ & $\begin{array}{c}16.7 \\
(5)\end{array}$ & 3.43 \\
\hline 31 & $\begin{array}{l}\text { I try to find out how to be a better learner } \\
\text { of Arabic. }\end{array}$ & - & $\begin{array}{l}6.7 \\
(2)\end{array}$ & $\begin{array}{c}16.7 \\
(5)\end{array}$ & $\begin{array}{l}53.3 \\
(16)\end{array}$ & $\begin{array}{c}23.3 \\
(7)\end{array}$ & 3.93 \\
\hline 32 & $\begin{array}{l}\text { I have clear goals for improving my } \\
\text { Arabic skills. }\end{array}$ & $\begin{array}{l}3.3 \\
(1)\end{array}$ & - & $\begin{array}{c}30.0 \\
(9)\end{array}$ & $\begin{array}{l}46.7 \\
(14)\end{array}$ & $\begin{array}{c}20.0 \\
(6)\end{array}$ & 3.80 \\
\hline 33 & $\begin{array}{l}\text { I look for opportunities to read as much } \\
\text { as possible in Arabic. }\end{array}$ & $\begin{array}{l}3.3 \\
(1)\end{array}$ & $\begin{array}{l}10.0 \\
(3)\end{array}$ & $\begin{array}{c}26.7 \\
(8)\end{array}$ & $\begin{array}{l}50.0 \\
(15)\end{array}$ & $\begin{array}{c}10.0 \\
(3)\end{array}$ & 3.53 \\
\hline 34 & $\begin{array}{l}\text { I plan my schedule so I will have enough } \\
\text { time to study Arabic. }\end{array}$ & $\begin{array}{l}6.7 \\
(2)\end{array}$ & $\begin{array}{l}10.0 \\
(3)\end{array}$ & $\begin{array}{l}43.3 \\
(13)\end{array}$ & $\begin{array}{l}33.3 \\
(10)\end{array}$ & $\begin{array}{l}6.7 \\
(2)\end{array}$ & 3.23 \\
\hline 35 & $\begin{array}{l}\text { I notice my Arabic mistakes and use that } \\
\text { information to help me do better. }\end{array}$ & - & - & $\begin{array}{l}16.7 \\
(5)\end{array}$ & $\begin{array}{l}70.0 \\
(21)\end{array}$ & $\begin{array}{c}13.3 \\
(4)\end{array}$ & 3.96 \\
\hline
\end{tabular}

It was found in Table 4 above that the mean of descriptive statistics for metacognitive strategies was ranged between 3.23 and 4.16 . To illustrate, the mean of 3.23 was belonged to the strategy "planning the schedule in order to have enough time to study Arabic" (item 34) and the mean of 4.16 was pertained to the strategy"paying attention when someone is speaking Arabic" (item 27).

Table 5

Affective strategies

\begin{tabular}{|c|c|c|c|c|c|c|c|}
\hline $\begin{array}{l}\text { Item } \\
\text { No. }\end{array}$ & Statement & $\begin{array}{c}\text { Never } \\
\% \\
(\mathbf{N}) \\
\end{array}$ & $\begin{array}{c}\text { Not true } \\
\% \\
(\mathrm{~N}) \\
\end{array}$ & $\begin{array}{c}\text { Somewhat } \\
\% \\
(\mathbf{N}) \\
\end{array}$ & $\begin{array}{c}\text { True } \\
\% \\
(\mathbf{N}) \\
\end{array}$ & $\begin{array}{c}\text { Always } \\
\% \\
\text { (N) }\end{array}$ & $\mathbf{M}$ \\
\hline & Affective Strategies: & & & & & & \\
\hline 36 & $\begin{array}{l}\text { I try to relax whenever I feel afraid of } \\
\text { using Arabic. }\end{array}$ & $\begin{array}{l}3.3 \\
(1)\end{array}$ & - & $\begin{array}{c}23.3 \\
(7)\end{array}$ & $\begin{array}{l}60.0 \\
(18)\end{array}$ & $\begin{array}{l}13.3 \\
(4)\end{array}$ & 3.80 \\
\hline 37 & $\begin{array}{l}\text { I give myself a reward or treat when I } \\
\text { do well in Arabic. }\end{array}$ & $\begin{array}{l}6.7 \\
(2)\end{array}$ & $\begin{array}{c}26.7 \\
(8)\end{array}$ & $\begin{array}{c}30.0 \\
(9)\end{array}$ & $\begin{array}{l}33.3 \\
(10)\end{array}$ & $\begin{array}{l}3.3 \\
(1)\end{array}$ & 3.00 \\
\hline 38 & $\begin{array}{l}\text { I write down my feelings in a language } \\
\text { learning diary. }\end{array}$ & $\begin{array}{l}30.0 \\
(9)\end{array}$ & $\begin{array}{l}40.0 \\
(12)\end{array}$ & $\begin{array}{l}13.3 \\
(4)\end{array}$ & $\begin{array}{l}13.3 \\
(4)\end{array}$ & $\begin{array}{l}3.3 \\
(1)\end{array}$ & 2.20 \\
\hline 39 & $\begin{array}{l}\text { I notice if I am tense or nervous when I } \\
\text { am studying or using Arabic. }\end{array}$ & $\begin{array}{l}3.3 \\
(1)\end{array}$ & $\begin{array}{c}26.7 \\
(8)\end{array}$ & $\begin{array}{l}26.7 \\
(8)\end{array}$ & $\begin{array}{c}30.0 \\
(9)\end{array}$ & $\begin{array}{c}13.3 \\
(4)\end{array}$ & 3.23 \\
\hline 40 & $\begin{array}{l}\text { I encourage myself to speak Arabic } \\
\text { even when I am afraid of making a } \\
\text { mistake. }\end{array}$ & - & $\begin{array}{l}3.3 \\
(1)\end{array}$ & $\begin{array}{c}23.3 \\
(7)\end{array}$ & $\begin{array}{l}56.7 \\
(17)\end{array}$ & $\begin{array}{l}6.7 \\
(5)\end{array}$ & 3.86 \\
\hline 41 & $\begin{array}{l}\text { I talk to someone else about how I feel } \\
\text { when I am learning Arabic. }\end{array}$ & - & $\begin{array}{l}6.7 \\
(2)\end{array}$ & $\begin{array}{l}33.3 \\
(10)\end{array}$ & $\begin{array}{l}46.7 \\
(14)\end{array}$ & $\begin{array}{c}13.3 \\
(4)\end{array}$ & 3.66 \\
\hline
\end{tabular}

Table 5 above revealed that among 6 items regarding affective strategies, the participants preferred to use the strategy "encouraging themselves to speak Arabic even they feel afraid of making a mistake" (item 40) with the highest frequency, with mean of 3.86. On the other side, they used the strategy "writing the feelings in a language learning diary" (item 38), the mean was 2.20 .

Table 6

Social strategies

\begin{tabular}{|c|c|c|c|c|c|c|c|}
\hline $\begin{array}{l}\text { Item } \\
\text { No. }\end{array}$ & Statement & $\begin{array}{c}\text { Never } \\
\% \\
(\mathrm{~N}) \\
\end{array}$ & $\begin{array}{c}\text { Not true } \\
\% \\
(\mathrm{~N}) \\
\end{array}$ & $\begin{array}{c}\text { Somewhat } \\
\% \\
(\mathrm{~N}) \\
\end{array}$ & $\begin{array}{c}\text { True } \\
\% \\
(\mathrm{~N}) \\
\end{array}$ & $\begin{array}{c}\text { Always } \\
\% \\
(\mathrm{~N}) \\
\end{array}$ & M \\
\hline & Social Strategies: & & & & & & \\
\hline \multirow[t]{2}{*}{42} & I ask questions in Arabic to other & 3.3 & 6.7 & 46.7 & 40.0 & 3.3 & 3.33 \\
\hline & students or native Arabic speakers. & (1) & (2) & (14) & (12) & (1) & \\
\hline \multirow[t]{2}{*}{43} & I practice Arabic with other students. & 6.7 & 3.3 & 43.3 & 40.0 & 6.7 & 3.36 \\
\hline & & (2) & (1) & (13) & (12) & (2) & \\
\hline 44 & I discuss Arabic lesson with my friends & 3.3 & 3.3 & 20.0 & 53.3 & 20.0 & 3.83 \\
\hline
\end{tabular}


Language Learning Strategies Applied by Arabic Learners: A Case-Study at Institute of Education,

\begin{tabular}{|c|c|c|c|c|c|c|c|}
\hline & & (1) & (1) & (6) & $(16)$ & (6) & \\
\hline 45 & I ask for help from Arabic speakers. & 6.7 & 3.3 & 16.7 & 60.0 & 13.3 & 3.70 \\
\hline 46 & I ask Arabic speakers to correct me & 3.3 & 3.3 & 33.3 & 46.7 & 13.3 & 3.63 \\
\hline \multirow[t]{2}{*}{47} & I try to learn about the culture of & 3.3 & 16.7 & 26.7 & 46.7 & 6.7 & 3.36 \\
\hline & Arabic speakers. & (1) & $(5)$ & $(8)$ & (14) & $(2)$ & \\
\hline 48 & If I do not understand something in & 3.3 & 6.7 & 16.7 & 50.0 & 23.3 & 3.83 \\
\hline
\end{tabular}

Finally, Table 6 above displayed that the frequency of using social strategies was range within the mean of 3.33-3.83. Certainly, the strategy "asking questions in Arabic to other students or native Arabic speakers" (item 42) was the least frequently used by the respondents, carrying the mean of 3.33. The most frequently used by them on the contrary (mean 3.83) were the strategy "discussing Arabic lesson with friends" (item 44) and "asking the other person to slow down or repeat something in Arabicif they not understand" (item 48).

Table7

Most Strategies Practiced

\begin{tabular}{|c|c|c|c|c|c|}
\hline Strategy categories & $\mathbf{N}$ & $\mathbf{M}$ & SD & Level of Use & No. in Rank \\
\hline Memory & 30 & 3.40 & .397 & Medium & 4 \\
\hline Compensation & 30 & 3.61 & .576 & Medium & 2 \\
\hline Affective & 30 & 3.29 & .500 & Medium & 6 \\
\hline Social & 30 & 3.58 & .590 & Medium & 3 \\
\hline Overall & & 3.49 & .397 & Medium & \\
\hline
\end{tabular}

As indicated in table 7 above that metacognitive strategies were the highest rank used by the respondents. The mean score was 3.74, followed by compensation strategies, with a mean of 3.61. This mean score (3.61) was slightly greater than the mean score of social strategies (3.58). Furthermore, memory strategies were ranked in the fourth highest mean (3.40) and cognitive strategies were in the fifth (3.35) relatively. Surprisingly, affective strategies were the least frequently use by the participants,possessingthe mean of 3.29.

While metacognitive strategies were found the most frequently used, affective strategies were the least frequently used by the participants involved in this study. These findings are consistent with those of the studies by Al-Buainain (2010) and Riazi (2007). The aforesaid studies focused on the use of language learning strategies (LLS) in learning English language as L2 of FL by a variety of participants. These results imply a mutual status of L2 or FL of these two languages (English and Arabic) in some countries, as well as similar strategies used by the learners of these two languages. On the contrary, some findings in this study appear to be contradictory with the results of the preliminary study by Oxford (1990), who argues that Asian students, in general, prefer to apply rote memorization rather than other forms in language learning process. Similarly, the study by Chapakiya (1996), who notes that Arabic students in Southern Thailand particularly were more concerned with memorizing Arabic words and grammar. In this case, one could argue that those students might refer to those who learn Arabic at pondokinstitution only, not university students. Furthermore, Riazi (2007) asserts that nowadays learning approaches of requiring memorization of factual knowledge have been minimized in non-Western countries, and more effective learning approaches such as analysis, synthesis, and evaluation of the instructional materials are incorporated in the teaching and learning of a target language.

\section{Conclusion}

LLS play a significant role in second language acquisition (SLA) as they assist learners to make learning easier, faster, more enjoyable, more self-directed, more effective and more transferable to new situation. Therefore, one-way to be successful in the target language is that students must be conscious of using certain language learning strategies. The more strategies learners use, the more successful they will be.Therefore, for those who tend to be successful in second or foreign language learning generally and Arabic learning specifically, should be familiar with all six categories of LLS and utilize them more consciously.

\section{References}

[1]. Oxford, R.L. (1990). Language learning strategies. What every teacher should know. Boston: Heinle and Heinle.

[2]. Meshyan, G., \& Hernandez, A. (2002). Is native language decoding decoding skill related to second language learning. Journal of Educational Psychology, 94(1), 14-22. 
[3]. SuerayaCheHarun, Ismail Sheikh Ahmad, ArifinMamat, and IsmaielHassanein Ahmed Mohamed. (2010). Understanding Arabic skill learning strategies among selected Malay learners: A case-study at the International Islamic University Malaysia (IIUM). Contemporary Issue in Education Research-Aug 2010. Volume 3, November 8.

[4]. Bremner, S. (1998). Language learning strategies and language proficiency: Investigating the relationship in Hong Kong. Asian Pacific Journal of Language Education, 1, 490-514.

[5]. O’Malley, J. M., Chamot, A. U., Manzanares, G. S., Kupper, L., \& Russo, R. P. (1985). Learning strategies used by beginning and intermediate ESL students. Language Learning, 35, 21-46.

[6]. Politzer, R. (1983). An exploratory study of self-reported language learning behaviors and their relation to achievement. Studies in Second Language Acquisition,6, 54-65.

[7]. AnidaAbd. Rahim.(2003). Strategipembelajaranbahasa Arab di kalanganpelajarMelayu [Arabic Language learning strategies of the Malay Students].Unpublished master's thesis, University of Malaya, Kuala Lumpur.

[8]. Burke, J. C. (2005).Arabic language study in the United States after 9/11. Muslim Education Quarterly, 22

[9]. DuerehMahamah. (1999). Al-akhta' fi istikhdamiaddhamairi al-Arabiyyahlada at-talabah bi Jamiah al-Amir SonkhlaFatani.Unpublished master's thesis, International Islamic University, Malaysia.

[10]. Doka, Hassan Mohamed. (2000). Attitude of Malay student toward learning Arabic as a foreign language. Unpublished dissertation, University of Malaya, Malaysia

[11]. Etea, S. (2009). The utilization of internet websites in teaching and learning Arabic in Southern Thailand Universities. Unpublished master's thesis, International Islamic University Malaysia.

[12]. Hobrom, A. I. (2004). Online resources and learner autonomy: A study of college-level students of Arabic. Unpublished doctorial dissertation, The University of Texas at Austin.Available online.Retrieved March 21, 2012.http://lib.utexas.edu/etd/d/2004/hobromd4198/hobromd4198.pdf

[13]. Qais Faryadi, Zainab Abu Bakar, \& Hamidah Maidinsah. (2007). Determining an effective interactive multimedia Arabic language courseware for Malaysian primary school children: An alternative paradigm for learning in the classroom. Online Submission, Paper presented at National Conference on software engineering \& computer systems (Kuantan, Malaysia, 2007). (ERIC Document Reproduction Service No.ED499175).

[14]. Wan Nordin Bin Wan Abdullah. (2006). Penggunaan ICT dalam pengajaran bahasa Arab di kalangan guru: Kajian di sebuah pusat bahasa Arab. Unpublished Master's thesis, University of Malaya, Malaysia.

[15]. Creswell, John.W.(2008). Educational research: Planning, conducting, and evaluating quantitative and qualitative research (Fifth edition). New Jersey: Pearson Merrill Prentice.

[16]. Robson, Colin (1997). Real World Research, A Resource for Social Scientists and Practitioner-Researchers. Oxford: Blackwell Publishers Ltd.

[17]. al-Buainain, H. (2010). Language learning strategies employing by English majors at Qatar University: Question and queries. Aisatic, Vol 4, November 2, December 2010.

[18]. Riazi, A. (2007). Language learning strategies use: perceptions of female Arab English majors. Foreign language Annual; Fall 2007; 40, 3; proquest education journals, p. 433.

[19]. ChapakiaCheuseng. (1996). Teaching Arabic as second language in Pattani, South of Thailand: Problems and Possibilities. Unpublished master's thesis, International Islamic University Malaysia. 